\title{
A comparison of echocardiographic and electron beam computed tomographic assessment of aortic valve area in patients with valvular aortic stenosis
}

\author{
Lieuwe H. Piers · Riksta Dikkers · René A. Tio $\cdot$ Maarten P. van den Berg • \\ Tineke P. Willems $\cdot$ Felix Zijlstra $\cdot$ Matthijs Oudkerk
}

Received: 16 October 2006/ Accepted: 17 January 2007 / Published online: 7 February 2007

(C) Springer Science+Business Media B.V. 2007

\begin{abstract}
The purpose of this study was to compare electron beam computed tomography (EBT) with transthoracic echocardiography (TTE) in determining aortic valve area (AVA). Thirty patients ( 9 females, 21 males) underwent a contrast-enhanced EBT scan (e-Speed, GE, San Francisco, CA, USA) and TTE within $17 \pm 12$ days. In end-inspiratory breath hold, a prospectively ecg-triggered scan was acquired with a beam speed of $50-100 \mathrm{~ms}$, a collimation of $2 \times 1.5 \mathrm{~mm}$ and an increment of $3.0 \mathrm{~mm}$. The AVA was measured with planimetry. A complete TTE study was performed in all patients, and the AVA was computed using the continuity equation. There was close correlation between AVA measured with EBT and AVA assessed with TTE $(r=0.60, P<0.01)$. The AVA measured with EBT was $0.51 \pm 0.46 \mathrm{~cm}^{2}$ larger than the AVA calculated with TTE measurements. EBT appeared to be a valuable non-invasive method to
\end{abstract}

L. H. Piers $(\bowtie) \cdot$ R. A. Tio · M. P. van den Berg .

F. Zijlstra

Department of Cardiology, University Medical

Centre Groningen, University of Groningen,

Hanzeplein 1, P.O. Box 30001, Groningen 9700 RB,

The Netherlands

e-mail: 1.h.piers@thorax.umcg.nl

R. Dikkers · T. P. Willems · M. Oudkerk

Department of Radiology, University Medical Centre Groningen, University of Groningen, Groningen,

The Netherlands measure the AVA. EBT measures the anatomical AVA, while with TTE the functional AVA is calculated, which explains the difference in results between the methods.

Keywords Aortic valve stenosis - Electron beam computed tomography · Echocardiography
Abbreviations
AVA Aortic valve area
AVC Aortic valve calcification
EBT Electron beam computed tomography
MRI Magnetic resonance imaging
TTE Transthoracic echocardiography

\section{Introduction}

Aortic valve sclerosis is common in the elderly population, affecting about $25 \%$ of adults over 65 years of age $[1,2]$. The presence of aortic sclerosis, with or without demonstrable haemodynamic obstruction, is associated with an increase of approximately $50 \%$ in the risk of cardiovascular death [3]. The timing of aortic valve replacement surgery has also an effect on mortality risk [4]. The indication for aortic valve replacement generally is based on haemodynamic 
variables, such as transvalvular pressure drop and the presence of symptoms. Significant reduction in the aortic valve area (AVA) is also an important indicator [4]. Hence, exact evaluation of the aortic valve and the AVA is necessary to make the best treatment decision.

Evaluation of aortic valve stenosis by means of transthoracic echocardiography (TTE) has gained widespread acceptance in clinical routine and pressure gradients across the aortic valve have shown to correlate well with invasive measurements $[5,6]$. Moreover, TTE is used to determine the functional AVA, using the continuity equation $[5,6]$. However, the reliability of TTE measurements depends heavily on image quality, which is influenced by aortic valve calcification and adequacy of the ultrasound window [7-9]. The results also depend on the patient's haemoglobin level, heart rate and left ventricular function [7]. Thus, invasive confirmation is often still necessary preceding valve replacement surgery. For this purpose, cardiac catheterization evaluating AVA by applying the Gorlin formula is available [10]. However, this method may not give reliable results, depending on cardiac function and aortic regurgitation [11]. Aforementioned techniques rely on and are affected by physiological parameters. Moreover, catheterization is an invasive procedure that may be associated with serious complications [12]. Therefore, new techniques are necessary to evaluate the severity and progression of aortic sclerosis. These new techniques could measure the anatomical AVA, which measurement is independent of physiological parameters.

Contrast-enhanced computed tomography is a useful technique to evaluate cardiac structures, like coronary arteries and aortic valves [13]. EBT, a non-mechanical computed tomography scan with a high temporal resolution (50-100 ms), is widely used to assess cardiac calcification, which is also an early marker of the aortic sclerotic process $[1,2]$. To assess cardiac calcification, administration of contrast agent to the patient is not necessary. Several studies have shown the usefulness of assessing the degree of aortic valve calcification (AVC) with EBT as a measure of the severity of aortic valve sclerosis [14-17]. However, these studies did not use EBT to measure the AVA. MacMillan et al. were the first to use contrast-enhanced EBT to measure the AVA [18]. They were able to determine AVA in six patients with known aortic sclerosis. We wished to compare EBT and TTE in order to compare the assessment of aortic stenosis with both methods. We hypothesize that EBT is an useful method to evaluate aortic stenosis; both in terms of AVA as well as AVC.

\section{Methods}

Between June 2004 and February 2006, patients with a known peak gradient $(\geq 30 \mathrm{mmHg}$ ) across their native aortic valve were invited to participate in the study during their control visit to the outpatient clinic of cardiology. All patients were under control for valvular aortic stenosis. The study was in accordance with principles that have their origin in the Declaration of Helsinki, and was approved by the local review board and all patients gave their informed consent. All patients were over 18 years of age, and had to be able to lie flat and hold their breath for $20 \mathrm{~s}$. Patients with congenital heart defects, renal insufficiency (serum creatinin $>120 \mu \mathrm{mol} / \mathrm{l}$ ), known contrast allergy or history of hyperthyroidism, thyroid cancer, Kahlers disease, myasthenia gravis, pheochromocytoma or mastocytosis were excluded. Patients with a body weight over $100 \mathrm{~kg}$ were also excluded because image quality of EBT is impaired in obese patients.

\section{Electron beam computed tomography}

Scans were performed with an EBT scanner (e-Speed, GE, San Francisco, CA, USA), in the dual $1.5 \mathrm{~mm}$ slice mode with an image acquisition time of 50 or $100 \mathrm{~ms}$ depending on patient size. First, non-enhanced EBT data were collected during end-inspiratory breath hold. The nonenhanced scan was ecg-triggered at $42 \%$ of the $\mathrm{R}-\mathrm{R}$ interval. A set of 40 continuous axial coupes were obtained from under the trachea bifurcation to the heart. An Agatston calcification score of the aortic valve was obtained [19]. Area and volume of calcifications were calculated and summated. The localization and severity of 
AVC was graded using a scale of 1 to 6 according to Bahler et al. [20].

Second, contrast-enhanced EBT data were collected during end-inspiratory breath hold. To achieve optimal contrast enhancement, bolus time was determined for each patient individually before EBT scanning. Bolus timing, using $20 \mathrm{ml}$ contrast agent, was done with the region of interest placed in the ascending aorta. The ecgtriggered multiphase scan was carried out with $120 \mathrm{ml}$ contrast agent (Iomeron $400^{\circledR}$, Bracco, Italy) followed by a saline bolus chaser. Contrast and saline bolus chaser were injected through a $18 \mathrm{G}$ venflon, placed in a cubital vein, with a flow rate of $4 \mathrm{ml} / \mathrm{s}$. Ecg-triggered multiphase scanning started at $5 \%$ of the $\mathrm{R}-\mathrm{R}$ interval, the first phase, and continued during systole till $50 \%$ of the R-R interval, the last phase. Depending on the patient's heart rate and beam speed, measurements were performed during 7 to 10 phases.

The data were transferred to a workstation (GE Advanced Workstation, GE Medical, Waukesha, WI, USA) and reviewed by 1 observer (R D), blinded from echocardiographic results. An axial view of the aorta valve was created with a double oblique view. One oblique axis was put trough the aortic valve in the coronal view of the heart, see Fig. 1(A). The second oblique axis was set in the created oblique view, see Fig. 1(B). The AVA was than measured in six planes at several levels in the created axial view of the aortic valve, see Fig. 1(B). The plane with the smallest AVA was selected and measured three times using planimetry, see Fig. 1(C). The mean AVA was assessed in this way for each phase. The phase with the maximal mean AVA was selected and was considered to be the AVA of concern. This AVA was compared to TTE findings.

\section{Transthoracic echocardiography}

A complete TTE study was performed in all patients by an experienced sonographer, blinded for EBT, following a standard procedure (GE Vingmed Ultrasound Vivid Five, GE Medical Systems, Waukesha, WI). The diameter of the left ventricular outflow tract was measured from the parasternal long-axis view. Flow velocity in the left ventricular outflow tract was assessed by pulse-wave Doppler from the apical 4 chamber approach. Flow velocity across the aortic valve was measured in the apical 5 chamber view. The AVA was computed using the continuity equation [5]. The presence of aortic regurgitation was determined from standard images, and quantified as the short-axis area of the regurgitant jet as a percentage to the short-axis area of the left ventricular outflow tract, moreover standard images of the left ventricle were obtained to evaluate left ventricular function and hypertrophy. Left ventricular function was based on estimated ejection fraction and wall motion, and was graded as being normal if the ejection fraction $\geq 60 \%$. Left ventricular dysfunction was mild if the ejection fraction was $45-60 \%$, moderate if the ejection fraction $30-45 \%$, or poor if the
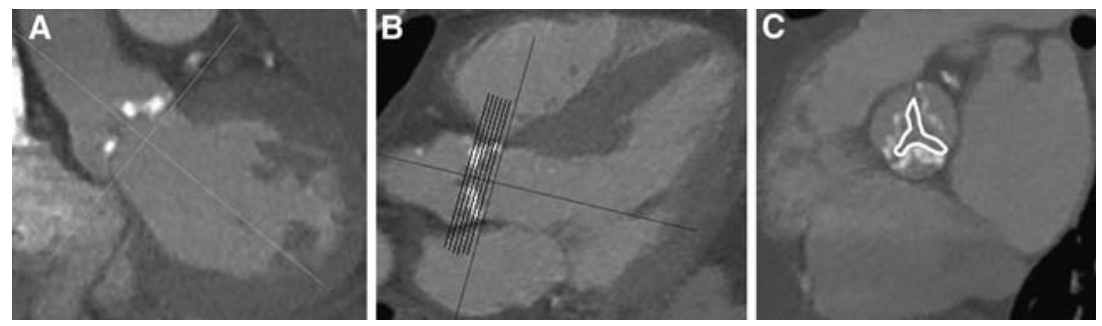

Fig. 1 All patients underwent electron beam computed tomography. (A) One oblique axis was put trough the aortic valve in the coronal view of the heart. Based on this orientation an oblique view was created. (B) The second oblique axis was set in the created oblique view. Based on this orientation an axial view of the aortic valve was made. The aortic valve area (AVA) was than measured in six planes at several levels in the created axial view of the aortic valve. (C) The AVA was measured three times at each level in each phase using planimetry. The mean AVA was assessed for each phase. The phase with the maximal mean AVA was selected and was considered to be the AVA of concern 
ejection fraction was $<30 \%$. To evaluate left ventricular hypertrophy, posterior wall and septal thickness were measured from the parasternal length axis.

\section{Statistical analysis}

For the measurement of the AVA by EBT, 3 manual tracings made by 1 observer (R D) were averaged. Results of continuous normally distributed variables are expressed as mean $\pm \mathrm{SD}$, results of continuous not normally distributed variables are expressed as median (range). The data were analyzed with the use of standard software (SPSS version 12.0.1, SPSS Institute, Chicago, IL, USA) on a PC. The Spearman's and Pearson's correlation coefficients (r) were used to establish the presence of linear relationships. To compare not normally distributed data, the Mann-Whitney U test was used. A Bland-Altman analysis was used to evaluate the agreement between the AVA assessed with EBT and TTE. A level of significance below 0,05 was defined as clinically significant.

\section{Results}

A total of 61 patients were screened for participation in the study. Nine females and 21 males (age $70.9 \pm 10.0$ years) were enrolled in the study. Eight patients were excluded because of renal insufficiency, 1 because of known contrast allergy, 1 because of body weight over $100 \mathrm{~kg}$, and 4 patients declined to undergo an EBT. Another 17 patients were excluded because the AVA could not be assessed by TTE.

In the study group, the mean peak pressure gradient across the valve was $64 \pm 21 \mathrm{mmHg}$. Ten patients had symptoms according to NYHA class I, 12 patients class II and 8 patients class III. Left ventricular end-diastolic diameter was $4.9 \pm 0.6 \mathrm{~cm}$, interventricular septal thickness was $1.2 \pm 0.3 \mathrm{~cm}$ and posterior wall thickness was $1.1 \pm 0.1 \mathrm{~cm}$. Ninety percent of the patients had signs of left ventricular hypertrophy on TTE. Twenty two patients (73\%) had normal left ventricular function, 6 patients $(20 \%)$ had mild and 2 patients $(7 \%)$ moderate left ventricular dysfunction. Mild aortic regurgitation was present in 8 patients $(27 \%)$ and moderate aortic regurgitation in 2 patients (7\%). The average time between TTE and EBT was $17 \pm 12$ days.

Aortic valve area

Mean AVA assessed with TTE was $0.99 \pm 0.31 \mathrm{~cm}^{2}$. The median AVA measured with EBT was $1.34(0.69-2.84) \mathrm{cm}^{2}$. There was a significant correlation between AVA measured with EBT and AVA assessed with TTE ( $r=0.60$, $P<0.01)$, as shown in Fig. 2. The AVA measured with EBT was on average $0.51 \pm 0.46 \mathrm{~cm}^{2}$ larger than the AVA assessed with TTE, which was confirmed with Bland-Altman analysis, see Fig. 2. The difference in AVA between EBT and TTE showed a not statistically significant trend to be larger in patients with mild aortic regurgitation than without aortic regurgitation (0.62 (0.23-1.22) $\mathrm{cm}^{2}$ and $0.33(-0.14-1.52) \mathrm{cm}^{2}$, respectively, $P=0.08)$. The difference in AVA did not differ between patients with normal left ventricular function and left ventricular dysfunction $\left(0.45(-0.14-1.52) \mathrm{cm}^{2}\right.$ and $0.43(-0.03-1.26)$ $\mathrm{cm}^{2}$, respectively, $\left.P=0.78\right)$

Aortic valve calcification

The median Agatston score of the aortic valve was 3363 (425-10230). There was a moderate, but significant, inverse correlation between the Agatston score of the aortic valve measured with EBT and AVA assessed with TTE $(r=-0.38$, $P=0.04)$, as shown in Fig. 3. In 17 patients with an AVA $<1.0 \mathrm{~cm}^{2}$ the aortic valve was more calcified than in 13 patients with an AVA $\geq 1.0 \mathrm{~cm}^{2}$ (Agatston score 3879 (119210230) and 2447 (425-6378), respectively, $P=0.04)$.

\section{Discussion}

EBT is an useful non-invasive method to evaluate aortic stenosis, both in terms of AVA and AVC. However, on average a larger AVA was measured with EBT than was assessed AVA with TTE. Indeed, this is not surprising since EBT 


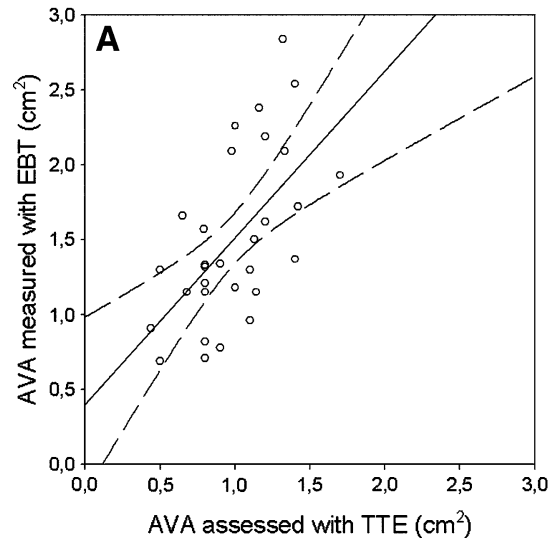

Fig. 2 By using electron beam computed tomography (EBT) planimetry the aortic valve area (AVA) can be measured. (A) AVA measured with EBT correlate well with those assessed with transthoracic echocardiography (TTE) $(r=0.60, P<0.01)$. (B) AVA measured with EBT

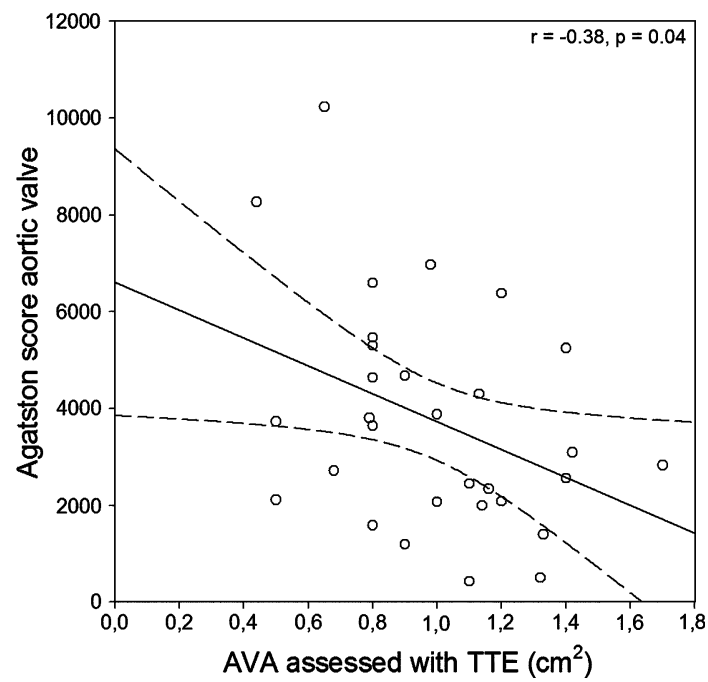

Fig. 3 Aortic valve calcification can be quantified with electron beam computed tomography using the Agatston score. The Agatston score correlates well with the aortic valve area (AVA) measured with transthoracic echocardiography $(\mathrm{TTE})(r=-0.38, P=0.04) .---=95 \%$ confidence interval

determines the anatomical AVA, which is expected to be greater than the functional AVA assessed with TTE. Because of calcifications the valvular leaflets are less flexible, as a result the AVA will be irregularly shaped, hence the AVA becomes haemodynamically less efficient. This results in a discrepancy between the functional and anatomical AVA.

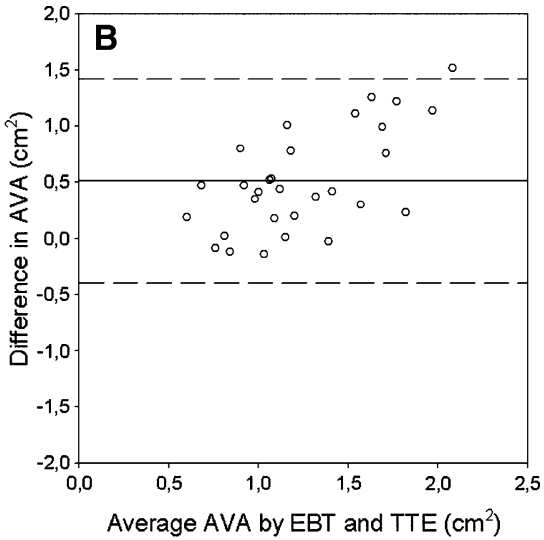

is larger on average than AVA assessed with TTE, as is confirmed with Bland-Altman analysis, exhibiting a mean difference of $0.51 \pm 0.46 \mathrm{~cm}^{2}$. $---=95 \%$ confidence interval

To our knowledge, the publication of MacMillan et al. is the first study investigating the usefulness of contrast-enhanced EBT to assess AVA [18]. In this study, 8 patients with known calcific aortic stenosis underwent EBT one day after cardiac catheterization. In six out of eight patients close agreement was found, within a $0.25 \mathrm{~cm}^{2}$ margin, between catheterization-derived AVA and AVA measured with EBT. In 1 patient the AVA could not be determined EBT and in 1 patient the AVA was overestimated by EBT with $0.60 \mathrm{~cm}^{2}$.

The results of our study are in line with those of MacMillan et al. Our study also showed that EBT measures larger AVA compared to TTE. The AVA is measured planimetrically with EBT, so it can be considered as the anatomical AVA. Our study as well as the study of MacMillan et al. compared this anatomical AVA to the functional AVA. This functional AVA is derived from haemodynamic characteristics of the aortic valve, (pressure changes and flow velocity changes) across the aortic valve. The functional AVA is supposed to yield the haemodynamic relevant AVA. However, using the continuity equation, the AVA is assumed to be circular, which does not reflect the actual morphology of the orifice of the stenotic valve, especially not in case of heavy calcification.

TTE is the principal diagnostic tool in routine clinical practice to assess the AVA, next to 
cardiac catheterization. To determine the AVA with TTE, the continuity equation is used $[5,6]$, which yields the functional AVA. However, this technique has its limitations. First, TTE is not suitable in every patient with aortic valve sclerosis. Poor sonographic windows and calcified deposits in the aortic valve and cardiac wall hamper the determination of the left ventricular outflow diameter. A small fault in the measurement of this diameter can seriously influence the calculated AVA, because this diameter is squared in the continuity equation. Moreover, if the ultrasound beam is not directed parallel to the velocity jet, peak transvalvular velocity may be underestimated. Hence the peak pressure gradient and AVA may be underestimated. Second, additional cardiac dysfunction can influence the outcome of TTE measurements. The flow velocity can be decreased in case of poor left ventricular function, even though there is an anatomical small AVA. This leads to an underestimation of the severity of the aortic stenosis. Furthermore, the severity of aortic stenosis can be overestimated in cases with concomitant aortic regurgitation. Consequently, the aortic valve stenosis is overestimated [7-9]. Our study also showed a larger difference in AVA between EBT and TTE in patients with additional mild aortic regurgitation EBT is not influenced by aortic regurgitation, therefore EBT is a more reliable technique, in those patients, to conclude about the severity of aortic stenosis.

The larger AVA measured by EBT compared to TTE and cardiac catheterization may be related to slice orientation. In order to get an axial view of the aortic valve, the image has to be rotated in two planes by the observer. If the selected axial view is not situated perpendicular to the aortic valve, the AVA is overestimated. Moreover, from this axial view the plane with the smallest AVA has to be selected. Although the temporal resolution of EBT is high, it is possible that the smallest AVA is situated between 2 planes. Hence, the AVA can be overestimated. Nevertheless, we addressed this problem by acquiring several slices at different levels of the aortic valve. This is necessary to minimize the potential of AVA overestimation because of imprecise localization.
In addition to the measurement of AVA, AVC can be quantified with EBT. Many studies have made clear that EBT is also effective in the evaluation of AVC [14-17, 21, 22]. The findings of these studies suggest that elevated AVC scores are a marker for the presence of aortic stenosis; our study underlined these findings. A diagnostic threshold value of 1000 Agatson units has a sensitivity of $93 \%$ to detect severe aortic stenosis (AVA $<1.0 \mathrm{~cm}^{2}$ ) according to Messika-Zeitoun et al. [16]. Rosenhek et al. concluded that the extent of AVC was a strong independent predictor of outcome [23]. In a study population of 126 patients with asymptomatic severe aortic stenosis, four-year event-free survival was $75 \%$ in patients with mild AVC, compared to $20 \%$ in patients with moderate or severe AVC.

As mentioned above, the grade of AVC is a predictor of outcome in patients with aortic stenosis. Although the echocardiographic severity of aortic stenosis is correlated to AVC score, the AVA can not be predicted from the AVC score. So, to evaluate the severity of aortic valve stenosis and to indicate whether aortic valve replacement surgery is necessary it is inevitable to measure AVA. In our study, we showed a significant correlation between the AVA measured with EBT and the severity of aortic stenosis assessed with TTE. However, further research is needed to validate EBT as a technique to evaluate the AVA.

Next to EBT, there are other non-invasive imaging techniques being evaluated for their value in AVA assessment. Alkadhi et al. compared 16 detector row computed tomography to tranesophageal echocardiography in the evaluation of AVA in 20 patients with and 20 patients without aortic stenosis [24]. They reported a mean difference of $0.06 \mathrm{~cm}^{2}$. Reant et al. performed a similar study using magnetic resonance imaging (MRI) [25]. They enrolled 39 patients with aortic stenosis and compared the AVA measured with MRI to tranesophageal echocardiography and cardiac angiography. Their results suggest good correlation between techniques; MRI and transesophageal echocardiography differed $0.01 \mathrm{~cm}^{2}$, MRI and cardiac angiography differed $0.06 \mathrm{~cm}^{2}$. Both studies show that noninvasive imaging techniques are a good possible 
alternative for the evaluation of aortic valve stenosis.

\section{Conclusion}

EBT appears to be valuable as a non-invasive method to assess the severity of aortic valve sclerosis. EBT holds the qualifications for accurate assessment of the anatomy, morphology and physiology of the aortic valve. However, further investigations will have to be done to verify whether EBT is a suitable non-invasive imaging technique for evaluation of aortic valve disease.

\section{References}

1. Lindroos M, Kupari M, Heikkila J, Tilvis R (1993) Prevalence of aortic valve abnormalities in the elderly: an echocardiographic study of a random population sample. J Am Coll Cardiol 21:1220-1225

2. Stewart BF, Siscovick D, Lind BK et al (1997) Clinical factors associated with calcific aortic valve disease. Cardiovascular Health Study. J Am Coll Cardiol 29:630-634. DOI:10.1016/S0735-1097(96)00563-3

3. Otto CM, Lind BK, Kitzman DW, Gersh BJ, Siscovick DS (1999) Association of aortic-valve sclerosis with cardiovascular mortality and morbidity in the elderly. N Engl J Med 341:142-147

4. Bonow RO, Carabello B, De Leon A Jr et al (1998) Guidelines for the management of patients with valvular heart disease: executive summary. A report of the American College of Cardiology/American Heart Association Task Force on Practice Guidelines (Committee on Management of Patients with Valvular Heart Disease). Circulation 98:1949-1984

5. Skjaerpe T, Hegrenaes L, Hatle L (1985) Noninvasive estimation of valve area in patients with aortic stenosis by Doppler ultrasound and two-dimensional echocardiography. Circulation 72:810-818

6. Otto CM, Pearlman AS, Comess KA, Reamer RP, Janko CL, Huntsman LL (1986) Determination of the stenotic aortic valve area in adults using Doppler echocardiography. J Am Coll Cardiol 7:509-517

7. Danielsen R, Nordrehaug JE, Vik-Mo H (1989) Factors affecting Doppler echocardiographic valve area assessment in aortic stenosis. Am J Cardiol 63:1107-1111. DOI:10.1016/0002-9149(89)90087/8

8. Geibel A, Gornandt L, Kasper W, Bubenheimer P (1991) Reproducibility of Doppler echocardiographic quantification of aortic and mitral valve stenoses: comparison between two echocardiography centers. Am J Cardiol 67:1013-1021. DOI:10.1016/00029149(91)90167-L
9. Bartunek J, De Bacquer D, Rodrigues AC, De Bruyne B (1995) Accuracy of aortic stenosis severity assessment by Doppler echocardiography: importance of image quality. Int J Card Imaging 11:97-104. DOI: 10.1007/BF01844707

10. Gorlin R, Gorlin SG (1951) Hydraulic formula for calculation of the area of the stenotic mitral valve, other cardiac valves, and central circulatory shunts. I. Am Heart J 41:1-29

11. Burwash IG, Dickinson A, Teskey RJ, Tam JW, Chan KL (2000) Aortic valve area discrepancy by Gorlin equation and Doppler echocardiography continuity equation: relationship to flow in patients with valvular aortic stenosis. Can J Cardiol 16:985992

12. Chambers J, Bach D, Dumesnil J, Otto C, Shah P, Thomas J (2004) Crossing the aortic valve in severe aortic stenosis: no longer acceptable? J Heart Valve Dis 13:344-346

13. Boxt LM (2005) CT of valvular heart disease. Int J Cardiovasc Imaging 21:105-113

14. Kaden JJ, Freyer S, Weisser G et al (2002) Correlation of degree of aortic valve stenosis by Doppler echocardiogram to quantity of calcium in the valve by electron beam tomography. Am J Cardiol 90:554-557. DOI:10.1016/S0002-9149(02)02537-7

15. Kizer JR, Gefter WB, deLemos AS, Scoll BJ, Wolfe ML, Mohler ER III (2001) Electron beam computed tomography for the quantification of aortic valvular calcification. J Heart Valve Dis 10:361-366

16. Messika-Zeitoun D, Aubry MC, Detaint D et al (2004) Evaluation and clinical implications of aortic valve calcification measured by electron-beam computed tomography. Circulation 110:356-362. DOI:10.1161/ 01.CIR.0000135469.82545.D0

17. Shavelle DM, Budoff MJ, Buljubasic N, et al (2003) Usefulness of aortic valve calcium scores by electron beam computed tomography as a marker for aortic stenosis. Am J Cardiol 92:349-53. DOI:10.1016/S00029149(03)00646-5

18. MacMillan RM, Rees MR, Lumia FJ, Maranhao V (1988) Preliminary experience in the use of ultrafast computed tomography to diagnose aortic valve stenosis. Am Heart J 115:665-671. DOI:10.1016/00028703(88)90818-6

19. Agatston AS, Janowitz WR, Hildner FJ, Zusmer NR, Viamonte M Jr., Detrano R (1990) Quantification of coronary artery calcium using ultrafast computed tomography. J Am Coll Cardiol 15:827-832

20. Bahler RC, Desser DR, Finkelhor RS, Brener SJ, Youssefi M (1999) Factors leading to progression of valvular aortic stenosis. Am J Cardiol 84:1044-1048. DOI:10.1016/S0002-9149(99)00496-8

21. Budoff MJ, Mao S, Takasu J, Shavelle DM, Zhao XQ, O'Brien KD (2002) Reproducibility of electron-beam CT measures of aortic valve calcification. Acad Radiol 9:1122-1127. DOI:10.1016/S1076-6332(03)80513-5

22. Pohle K, Dimmler A, Feyerer R et al (2004) Quantification of aortic valve calcification with electron beam tomography: a histomorphometric validation study. Invest Radiol 39:230-234 
23. Rosenhek R, Binder T, Porenta G et al (2000) Predictors of outcome in severe, asymptomatic aortic stenosis. N Engl J Med 343:611-617

24. Alkadhi H, Wildermuth S, Plass A et al (2006) Aortic Stenosis: Comparative Evaluation of 16 -Detector Row CT and Echocardiography. Radiology 240:47-55
25. Reant P, Lederlin M, Lafitte S et al (2006) Absolute assessment of aortic valve stenosis by planimetry using cardiovascular magnetic resonance imaging: Comparison with transoesophageal echocardiography, transthoracic echocardiography, and cardiac catheterisation. Eur J Rad 59:276-283 